\title{
Investigations on the method for the estimation of impulse response using a rectangular pulse
}

\author{
Chun-Duck Kim,* Masato Abe,** and Ken'iti Kido* \\ * Research Center for Applied Information Sciences, Tohoku University, \\ 2-1-1, Katahira, Sendai, 980 Japan \\ **Education Center for Information Processing, Tohoku University, \\ Kawauchi, Sendai, 980 Japan
}

(Received 8 September 1983)

\begin{abstract}
This paper proposes a method to measure the impulse response of a system with long reverberation using a rectangular pulse as the source signal. The progress of digital device has provided us with various useful digital equipments such as a howling-cancel system. To realize the system, it is necessary to estimate the impulse response. A response to a pulsive signal has been used, for example, to estimate the reverberation time. However, since the pulsive signal is not equal to the impulse, cross-spectral technique is necessary to estimate the impulse response, and much computation time is required to compute many discrete Fourier transforms. On the other hand, we propose a method using a rectangular pulse with a finite amplitude. Under some conditions developed in this paper, the response of the system to the rectangular pulse can be approximately equal to the impulse response. Therefore, it is not necessary to compute Fourier transforms, and the method requires the least amount of computations. We describe first the conditions to estimate the impulse response accurately using a rectangular pulse. Next, an index is introduced to evaluate the accuracy of the estimated impulse response. Using this index, the conditions are investigated in detail by computer simulations. Actual room experiments are also carried out to show that the evaluation method by the index is actually effective in practical cases, where there are external noises.
\end{abstract}

PACS number: 43. 60. - c, 43. 45. Bk, 45. 55. $\mathrm{Br}$

\section{INTRODUCTION}

Several authors have investigated methods to estimate the impulse response of a system with long reverberation such as that of an actual room. The conventional cross-spectral technique ${ }^{1)}$ requires too much computation time because a considerable number of Fourier transforms have to be computed. Aoshima's method ${ }^{2)}$ improves the signal to noise ratio, but it is still necessary to compute a considerable number of Fourier transforms to estimate the impulse response. The method using a pulsive signal such as a rectangular pulse has been used for the estimation of decay curve of reverberation. However, even if a pulsive signal is used, the impulse response should be estimated by the cross-spectral technique, because the pulsive signal is not exactly the same as an impulse. Thus, even with this method, many computations are required for the estimation.

Here in this paper, we describe a method for the estimation of impulse response using a rectangular pulse. In the method, the response of a system to the rectangular pulse can be approximately equal to the impulse response of the system under some conditions developed in this paper. Therefore, this method requires the least number of computations.

The conditions can be satisfied by adjusting following 3 factors: (1) pulse width, (2) pulse amplitude, and (3) the timing relation between the pulse and the sampling time for $\mathrm{A} / \mathrm{D}$ conversion. For the first 
factor, it is well-known that the pulse width should be shorter for a better accuracy in the estimation of an impulse response. However, the effect of the pulse width on the accuracy of the estimated impulse response has not been evaluated up to now. For the second factor, it is not well-known that multiplications by an adequate coefficient are necessary to obtain an impulse response. For the third factor, the effect of the timing relation between the rectangular pulse and the sampling time on the accuracy of the estimated impulse response has not been investigated. In this paper we describe first the way to estimate the impulse response using a rectangular pulse with a finite amplitude. Next, the effect of the 3 factors on the accuracy is investigated using an index introduced in this paper. In the case that the estimated impulse response is used for digital echo cancellation in telephone lines, ${ }^{3)}$ the effect of the third factor is as big as those of the 2 former factors especially in the higher frequency range. Unless the third factor is taken into consideration, it is necessary to compensate the illegal timing relation using a method such as the Fourier transform.
This paper introduces first the index to evaluate the accuracy of estimated impulse response, and then discusses the effect of the 3 factors on the accuracy of the estimated impulse response.

\section{EVALUATION METHOD FOR ACCURACY OF ESTIMATED IMPULSE RESPONSE}

The convolution between an input signal and the estimated impulse response should correspond to the actual response of the system to the same input signal. Therefore, the power of the difference between the measured and the computed response to the input signal may be used for the evaluation of the accuracy of the estimated impulse response. Since we can set the condition that a very high signal to noise ratio can be achieved for the input signal, the power of the difference signal depends on the following 3 parameters: (1) power level of the input signal, (2) power spectrum of the input signal, and (3) noise mixed to the response of a system. Taking the above mentioned 3 parameters into consideration, the evaluation index $Q$ is introduced as follows:

$$
\begin{aligned}
Q & =10 \log _{10} \frac{\overline{\mid \text { computed response }- \text { measured response }\left.\right|^{2}}}{\overline{\mid \text { measured response }\left.\right|^{2}}} \\
& =10 \log _{10} \frac{\overline{|x(p) * \hat{h}(p)-y(p)|^{2}}}{\overline{|y(p)|^{2}}} \\
& =10 \log _{10} \frac{\frac{|x(p) *\{\hat{h}(p)-h(p)\}-n(p)|^{2}}{|x(p) * h(p)+n(p)|^{2}}}{\overline{\mid x(p)}}
\end{aligned}
$$

where

$x(p)$ : input signal,

$\hat{h}(p)$ : estimated impulse response,

$y(p)$ : measured output signal,

$h(p)$ : accurate impulse response, and

$n(p)$ : external noise.

In Eq. (1), * denotes the convolution integral, and overscore means the average value.

When a white noise, which has no correlation with the external noise $n(p)$, is used as an input signal $x(p)$, Eq. (1) can be expressed as follows:

$$
Q=10 \log _{10} \frac{\overline{|x(p) *\{\hat{h}(p)-h(p)\}|^{2}}+\overline{|n(p)|^{2}}}{\overline{|x(p) * h(p)|^{2}+|n(p)|^{2}}} .
$$

input signal, is neglected in Eq. (1') since the index $Q$ is the ratio of the power of difference signal to that of the measured response. We can use the most adequate signal as the input signal for the second parameter. We used a white noise as the input signal because the white noise has a flat power spectrum. For the third parameter, we can employ the same input signal to compare the accuracy of the estimated impulse responses obtained by different conditions. The index $Q$ directly reflects the difference of accuracy of the estimated impulse responses, since the signal to noise ratio is the same in all cases. However, even if an exact impulse response is obtained, the value of index $Q$ representing the accuracy is less than the inverse of the signal to noise ratio.

The first factor, the effect of a power level of an 


\section{D. KIM et al.: ESTIMATION OF IMPULSE RESPONSE USING A RECTANGULAR PULSE}

\section{ESTIMATION OF IMPULSE RESPONSE USING RECTANGULAR PULSE}

The observed output signal of a linear system is the impulse response of the system when the input signal is a unit impulse and no noise is mixed. However, the unit impulse cannot be realized in an actual physical system because the range of linearity is limited to a certain value and an impulse of infinite amplitude cannot be passed through the system. As a consequence, we have to use a pulse with a finite amplitude and some width as the input signal instead of the unit impulse.

Hereafter, we discuss the method for the estimation of an impulse response using a rectangular pulse with a finite amplitude and some width. Since the amplitude of the pulse is fixed at a certain value, the energy of the pulse is proportional to the pulse width. The pulse width is required to be extremely long to achieve a high signal to noise ratio. On the other hand, the pulse width is required to be as short as possible to make the frequency spectrum flat in the necessary frequency range.

The spectrum of the rectangular pulse shown in Fig. 1 (a) is expressed as follows:

$$
\begin{aligned}
R(f) & =\int_{a-\tau / 2}^{a+\tau / 2} A \exp (-j 2 \pi f t) d t \\
& =A \tau \frac{\sin (\pi f \tau)}{\pi f \tau} \exp (-j 2 \pi f a),
\end{aligned}
$$

where

$\pi$ : ratio of the periphery of circle to diameter,

$f$ : frequency,

$a:$ the time of pulse center, and

$\tau$ : pulse width (duration of rectangular pulse). The spectrum of the rectangular pulse is shown in Fig. 1 (b). The spectrum of rectangular pulse has zero points at the frequencies $1 / \tau, 2 / \tau, 3 / \tau, \ldots$, and the spectrum is not flat.

When the frequency range of the signal is limited to $f_{m}$, the sampling period $T$ should be shorter than $1 / 2 f_{m}$. By normalizing the relation between the pulse width and the sampling period $T$, the magnitude of the power spectrum is expressed as a function of the ratio $\tau / T$. Figure 2 shows the normalized power spectra of the rectangular pulses for 5 values of the ratio $\tau / T$. It is shown in the figure that the pulse width should be shorter than a half of the sampling

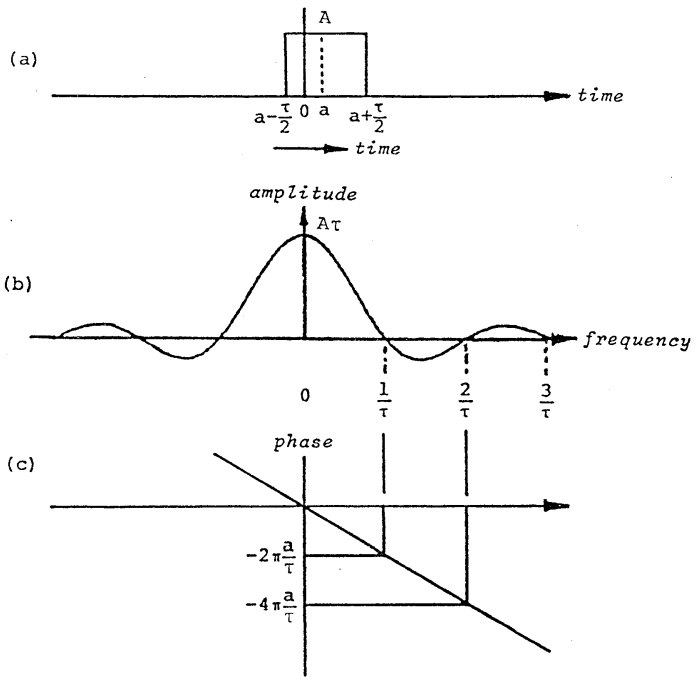

Fig. 1 Spectrum of a rectangular pulse. (a) relation between a rectangular pulse and the sampling timing, (b) magnitude of the spectrum, (c) phase of the spectrum.

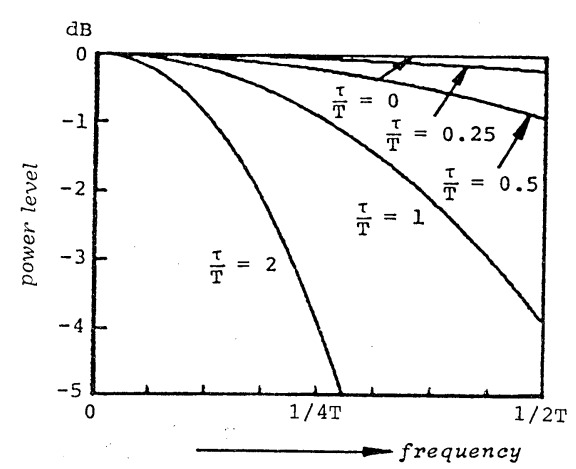

Fig. 2 Normalized power spectra of the rectangular pulse. $\tau$ : duration of the rectangular pulse, $T$ : sampling period.

period if the maximum decrease in the power spectrum is required to be less than $1 \mathrm{~dB}$. It is also seen that the pulse width can be the same as the sampling period if a decrease of $3.9 \mathrm{~dB}$ at the maximum frequency range is allowed.

The shorter source pulse width produces the flatter spectrum, but causes a deterioration in the signal to noise ratio. To improve the signal to noise ratio, we can superpose many responses by lining up the initial point of the response by a trigger pulse synchronized with the sampling pulse. It is well-known 
that the improvement in the signal to noise ratio has the following relation ${ }^{4}$ with the number of superposition $M$ :

Improvement in $\mathrm{S} / \mathrm{N}=10 \log _{10} M . \quad[\mathrm{dB}]$

The interval between the adjacent source pulses should be longer than the reverberation time of the transmission system to avoid the overlap of the responses.

When a computer is used to estimate the impulse response, computations should be carried out after A/D conversion. Then, the timing relation between the pulse and the sampling time as well as the width and the amplitude of the rectangular pulse, should also be investigated.

By using a simple model, we now discuss the degree to which the accuracy of an estimated impulse response is affected by the following 3 factors: (1) pulse width, (2) pulse amplitude, and (3) the timing relation between the pulse and the sampling time for A/D conversion.

Two sequences are made from an analogue signal $x(t)$ whose frequency range is limited to $f_{m} \mathrm{~Hz}$. The first sequence $x_{p}, p=0,1,2, \ldots, 2 P-1$ is made from $x(t)$ by sampling at the rate of $2 f_{x}$, where $f_{x}>f_{m}$. The second sequence $x_{n}^{\prime}, n=0,1,2, \ldots, 2 P N-1$ is made from the same signal by sampling at the rate of $2 N f_{x}$, where $N \gg 1$ is an integer. Under the condition that $\Delta T \rightarrow 0$ and $N \Delta T \neq 0$, the first sequence $x_{p}$ means the $\mathrm{A} / \mathrm{D}$ converted signal sampled at the rate of $N \Delta T$, and the second sequence $x_{n}^{\prime}$ means the analogue signal. The total numbers of the first and the second sequences are $2 P$ and $2 P N$, respectively. The relations between the sampling times and the numbers are shown in Fig. 3 (a) and in the following:

The sequence $x_{p}: \quad x_{p}=x(p N \Delta T)$,

The sampling period for $x_{p}$ :

$$
T=N \Delta T=1 / 2 f_{x},
$$

The sequence $x_{n}^{\prime}: \quad x_{n}^{\prime}=x(n \Delta T)$,

The sampling period for $x_{n}^{\prime}: \Delta T=1 / 2 N f_{x}$, and

The duration of sequences: $2 P T=2 P N \Delta T$.

The $2 P$ points discrete Fourier transforms (DFT) of $x_{p}$ and $2 P N$ points DFT of $x_{n}^{\prime}$ and their inverse transforms (IDFT) are expressed as follows:

$$
\left\{\begin{array}{l}
X_{k}=\sum_{p=0}^{2 P-1} x_{p} \exp \left(-j 2 \pi \frac{p k}{2 P}\right), \\
x_{p}=\frac{1}{2 P} \sum_{k=0}^{2 P-1} X_{k} \exp \left(j 2 \pi \frac{p k}{2 P}\right),
\end{array}\right.
$$

and

$$
\left\{\begin{array}{l}
X_{m}^{\prime}=\sum_{n=0}^{2 P N-1} x_{n}^{\prime} \exp \left(-j 2 \pi \frac{n m}{2 P N}\right), \\
x_{n}^{\prime}=\frac{1}{2 P N} \sum_{m=0}^{2 P N-1} X_{m}^{\prime} \exp \left(j 2 \pi \frac{n m}{2 P N}\right) .
\end{array}\right.
$$

The discrete frequencies $k$ and $m$ correspond to the

(a)

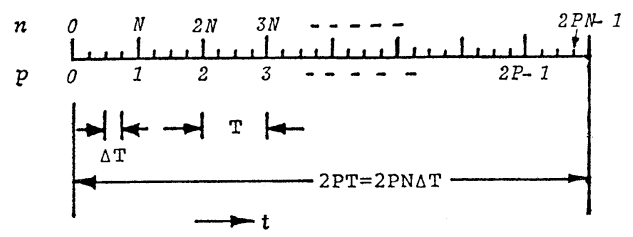

(b)

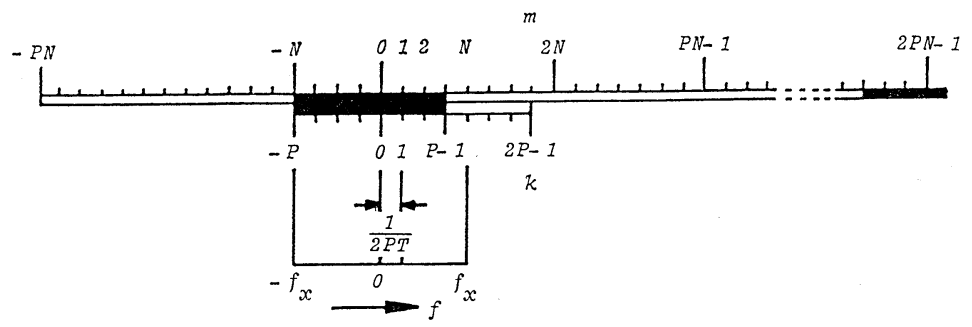

Fig. 3 Relation between the sampling period and the digitalized signals. 


\section{D. KIM et al.: ESTIMATION OF IMPULSE RESPONSE USING A RECTANGULAR PULSE}

analogue frequencies $k / 2 P N \Delta T$ and $m / 2 P N \Delta T$, respectively, since the durations of the sequences are equal to $2 P N \Delta T$ in both cases. The relations between the analogue frequency and the discrete frequencies $k$ and $m$ are illustrated in Fig. 3 (b).

The values of $k$ and $m$ are positive in Eqs. (5) and (6), but those exceeding $P$ and $P N$ correspond to the negative analogue frequencies due to the periodicity in DFT. The DFT's of $x_{p}$ and $x_{n}^{\prime}$ with negative subscript can be expressed as follows:

$$
\begin{gathered}
x_{p}=\frac{1}{2 P} \sum_{k=-P}^{P-1} X_{k} \exp \left(j 2 \pi \frac{p k}{2 P}\right), \\
x_{n}^{\prime}=\frac{1}{2 P N} \sum_{m=-P N}^{P N-1} X_{m}^{\prime} \exp \left(j 2 \pi \frac{n m}{2 P N}\right),
\end{gathered}
$$

where

$$
\begin{aligned}
& X_{-i}=X_{2 P-i}, \\
& X_{-i}^{\prime}=X_{2 P N-i}^{\prime} .
\end{aligned}
$$

The range of summation in Eq. (8) can be decreased since the frequency range of $x(t)$ is limited to $f_{m}<f_{x}=1 / 2 N \Delta T=P / 2 P N \Delta T$. Then, Eq. (8) can be rewritten as follows:

$$
x_{n}^{\prime}=\frac{1}{2 P N} \sum_{m=-P}^{P-1} X_{m}^{\prime} \exp \left(j 2 \pi \frac{n m}{2 P N}\right) .
$$

Since $x_{n}^{\prime}$ and $x_{p}$ are the sampled values of the identical wave, the following relation holds:

$$
x_{n}^{\prime}=x_{p}, \quad n=p N .
$$

From the relations shown in Eq. (4) and in Fig. 3 (b), Eq. (11) can be rewritten as follows:

$$
\begin{aligned}
x_{p N}^{\prime} & =\frac{1}{2 P N} \sum_{m=-P}^{P-1} X_{m}^{\prime} \exp \left(j 2 \pi \frac{p N m}{2 P N}\right) \\
& =\frac{1}{N} \frac{1}{2 P} \sum_{m=-P}^{P-1} X_{m}^{\prime} \exp \left(j 2 \pi \frac{p m}{2 P}\right) .
\end{aligned}
$$

Finally, the following relation is established from Eqs. (7), (10) and (12).

$$
X_{k}=\frac{1}{N} X_{k}^{\prime} .
$$

Equation (13) means that the DFT of the $P$ points sequence sampled at the sampling period of $N \Delta T$ is $1 / N$ in magnitude of that of the $P N$ points sequence sampled at the sampling period of $\Delta T$.

If the frequency range of the transfer function of the system is also limited lower than $f_{m}$, the following

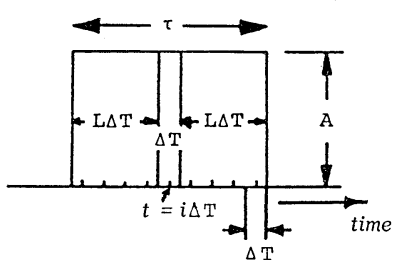

Fig. 4 Form of the rectangular pulse to be used for estimating the impulse response.

relation can be obtained in the same manner:

$$
H_{k}=\frac{1}{N} H_{k}^{\prime}, \quad-P \leq k<P,
$$

where $H_{k}$ and $H_{k}^{\prime}$ are the DFT's of the sequences of impulse responses $h_{p}=h(p N \Delta T)$ and $h_{n}^{\prime}=h(n \Delta T)$, respectively.

Let us consider that the rectangular pulse $v_{n}^{\prime}$ shown in Fig. 4 is the source signal for the estimation of impulse response. The amplitude of the pulse is $A$, and the duration $\tau$ is $(2 L+1) \Delta T$, where $L \gg 1$ is an integer. The sampling starts at $t=0$ and the midpoint of the pulse is at $t=i \Delta T$, where $i$ is an integer. The response of the system to the rectangular pulse $v_{n}^{\prime}$ is represented by $y_{n}^{\prime}$ as follows:

$$
y_{n}^{\prime}=v_{n}^{\prime} * h_{n}^{\prime} \text {. }
$$

Using the same relation explained in Eqs. (13) and (14), the DFT $Y_{k}$ of the sequence $y_{p}$ obtained by picking up every $N$ samples is expressed as

$$
\begin{aligned}
Y_{k} & =\frac{1}{N} Y_{k}^{\prime} \\
& =\frac{1}{N} V_{k}^{\prime} H_{k}^{\prime},
\end{aligned}
$$

where $Y_{k}^{\prime}$ is the DFT of $y_{n}^{\prime}$ and $V_{k}^{\prime}$ is the DFT of $v_{n}^{\prime}$ as follows:

$$
\begin{aligned}
V_{k}^{\prime}= & \sum_{p=i-L}^{i+L} A \exp \left(-j 2 \pi \frac{p k}{2 P N}\right) \\
= & A \exp \left(-j 2 \pi k \frac{i-L}{2 P N}\right) \\
& \times \frac{1-\exp \{-j 2 \pi(2 L+1) k / 2 P N\}}{1-\exp (-j 2 \pi k / 2 P N)} .
\end{aligned}
$$

In the actual case, the relations that $N \rightarrow \infty, L \rightarrow \infty$, $T \rightarrow 0, N \Delta T \rightarrow T,(2 L+1) \Delta T \rightarrow \tau$ and $-P \leq k<P$ are valid since the rectangular pulse $v_{n}^{\prime}$ is an analogue signal. Then, the following relations are obtained: 


$$
\begin{aligned}
& \exp \{j 2 \pi(2 L+1) k / 2 P N\} \\
& \fallingdotseq \exp \{j 2 \pi(2 L k) / 2 P N\} \\
& \exp (j 2 \pi k / 4 P N) \fallingdotseq 1
\end{aligned}
$$

and

$$
\sin (2 \pi k / 4 P N) \fallingdotseq 2 \pi k / 4 P N
$$

Using Eq. (18), Eq. (17) is expressed as

$$
\begin{aligned}
V_{k}^{\prime}= & A \exp (-j 2 \pi i k / 2 P N) \\
& \times \frac{\exp (j 2 \pi L K / 2 P N)-\exp \{-j 2 \pi(L+1) k / 2 P N\}}{\exp (j 2 \pi k / 4 P N)-\exp (-j 2 \pi k / 4 P N)} \\
& \times \exp (j 2 \pi k / 4 P N) \\
\fallingdotseq & A \exp (-j 2 \pi i k / 2 P N) \frac{\sin (2 \pi L k / 2 P N)}{\sin (2 \pi k / 4 P N)} \\
\doteqdot & 2 L A \exp (-j 2 \pi i k / 2 P N) \frac{\sin (2 \pi L k / 2 P N)}{2 \pi L k / 2 P N} .
\end{aligned}
$$

In our proposed method, the DFT $Y_{k}$ in Eq. (16) should be the same as the transfer function $H_{k}^{\prime}$. Hence, the following equation is obtained from Eqs. (16) and (19) as

$$
\frac{2 L A}{N} \frac{\sin (2 \pi L k / 2 P N)}{2 \pi L k / 2 P N} \exp (-j 2 \pi i k / 2 P N) \doteqdot 1 \text {. }
$$

It is clear from Fig. 4 and Eq. (4) that Eq. (20) is valid under the following three conditions:

(1) The duration of rectangular pulse is short so that

$$
\frac{\sin (2 \pi L k / 2 P N)}{2 \pi L k / 2 P N} \fallingdotseq 1
$$

(2) $2 \pi i k / 2 P N \fallingdotseq 0$,

and

(3) $A=N / 2 L \doteqdot N \Delta T /(2 L+1) \Delta T=T / \tau$.

Unless the three conditions expressed in Eqs. (21), (22) and (23) are satisfied, the accuracy of estimated impulse response is decreased. The condition expressed in Eq. (21) cannot be satisfied for all the frequency components because the rectangular pulse has some width.

To satisfy the condition expressed in Eq. (22), $i$ should be sufficiently smaller than $N$. This means that the center of the rectangular pulse just coincides with the sampling time for $\mathrm{A} / \mathrm{D}$ conversion. In the contrary case, for example, in the case that the width of rectangular pulse is the same as a half of the sampling period of $A / D$ conversion and the sampling time shifts from the center of the rectangular pulse by a quarter of the pulse width, the value $i / N$ in Eq. (20) is equal to $1 / 8$. Because of the relation that $-P \leq k<P$, the value of the third factor in Eq. (20) varies from $\exp (-j \pi / 8)$ to $\exp \{-j \pi(P-1) / 8 P\}$. Therefore, the condition expressed in Eq. (22) is necessary to estimate accurately the impulse response.

If the actual amplitude $A^{\prime}$ of the rectangular pulse is different from the value $A$ given in Eq. (23) for any reason, the following constant $C$ should be multiplied to the estimated impulse response:

$$
C=N / 2 L A^{\prime}=T / \tau A^{\prime} \text {. }
$$

Using this coefficient $C$, the amplitude of the source rectangular pulse is set to an arbitrary value, for which the peak value does not exceed the range of linearity.

\section{COMPUTER SIMULATION}

Though it is found that accuracy is decreased using a rectangular pulse as the source signal instead of impulse, the decreased amount of the accuracy is, however, still unknown. Hence, we examine the accuracy of the estimated impulse response by computer simulation.

The pulse train shown in Fig. 5 is used as the source pulse. The source rectangular pulse $v_{n}$ is assumed to be composed of $(2 L+1)$ pulses whose magnitude is determined by Eq. (23) and the $(L-i+1)$-th pulse was set at the sampling time.

The sequence of the impulse response of the room in the computer simulation is composed of 2,048 samples and is expressed by $h_{n}, n=1,2,3, \ldots$, where the sampling period is $T=N \Delta T$.

The response $\hat{h}_{n}^{\prime}$ of the room to the rectangular

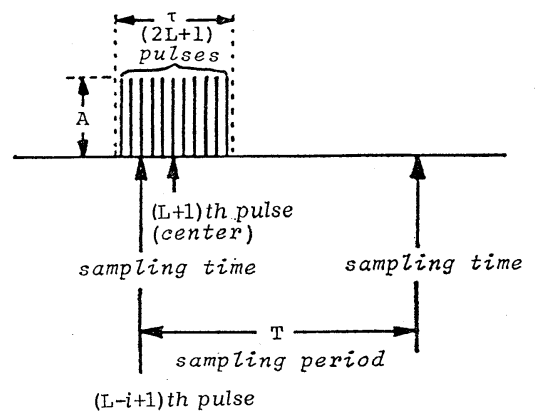

Fig. 5 Relation between the rectangular pulse and the sampling period for computer simulation. 


\section{D. KIM et al.: ESTIMATION OF IMPULSE RESPONSE USING A RECTANGULAR PULSE}

pulse of duration $(2 L+1) \Delta T$ can be expressed by the convolution between the source sequence $v_{n}$ and the impulse response $h_{n}^{\prime}$ as

$$
\hat{h}_{n}^{\prime}=v_{n}^{\prime} * h_{n}^{\prime} \text {. }
$$

To obtain the sequence of the impulse response by picking up at the sampling period of $N \Delta T$, the sequence $\hat{h}_{n}^{\prime}$ is needed to pass through an antialiasing filter to eliminate frequency components higher than $1 / 2 N \Delta T$. The response in Eq. (25) is now reduced to

$$
\tilde{h}_{n}^{\prime}=\hat{h}_{n}^{\prime} * h_{L n}^{\prime}=v_{n}^{\prime} * h_{n}^{\prime} * h_{L n}^{\prime},
$$

where $h_{L n}^{\prime}$ denotes the impulse response of the antialiasing filter.

A sequence $h_{p}$ is then composed by picking up every $N$ samples from the response $\tilde{h}_{n}^{\prime}$ as follows:

$$
h_{p}=\tilde{h}_{p N}^{\prime} \text {. }
$$

$h_{p}$ is the impulse response of the room to be obtained experimentally.

Next, we compute the index $Q$ representing the accuracy of estimated impulse response. A sequence $x_{n}^{\prime}$ composed of random numbers is first generated. The mean value and the standard deviation of $x_{n}^{\prime}$ are set to be 0 and 1.0, respectively. The sequence $x_{n}^{\prime}$ also passes through the anti-aliasing filter as follows:

$$
\tilde{x}_{n}^{\prime}=x_{n}^{\prime} * h_{L n}^{\prime} .
$$

The sequence $x_{p}$ of the source signal to be obtained experimentally is made from $\widetilde{x}_{n}^{\prime}$ by picking up every $N$ samples as

$$
x_{p}=\tilde{x}_{p N}^{\prime} \text {. }
$$

On the other hand, the response $z_{n}^{\prime}$ of the system to the input signal $x_{n}^{\prime}$ is written as

$$
z_{n}^{\prime}=x_{n}^{\prime} * h_{n}^{\prime} \text {. }
$$

To make the sequence with the sampling period of $N \Delta T, z_{n}^{\prime}$ should pass through anti-aliasing filter. The actual sequence $z_{p}$ to be obtained experimentally is expressed as follows:

$$
z_{p}=\tilde{z}_{p N}^{\prime}=\left(z_{n}^{\prime} * h_{L n}^{\prime}\right), \quad n=p N .
$$

On the other hand, the estimated response $\hat{z}_{p}$ to the input sequence $x_{p}$ is the convolution between $x_{p}$ and the estimated impulse response $h_{p}$ :

$$
\hat{z}_{p}=x_{p} * h_{p} .
$$

Subtracting $z_{p}$ from $\hat{z}_{p}$, the residual signal $y_{p}$ is initial point of sampling time/sampling time

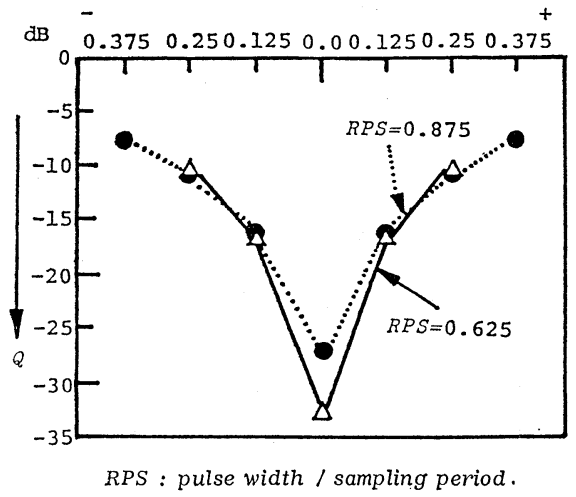

Fig. 6 Relation between the accuracy of the estimation and the shift of the center of the pulse from the sampling time.

defined as

$$
y_{p}=\hat{z}_{p}-z_{p} .
$$

Using $y_{p}$ and $z_{p}$, the evaluation index $Q$ is expressed as follows:

$$
\begin{aligned}
Q & =10 \log _{10} \frac{\overline{\left|\hat{z}_{p}-z_{p}\right|^{2}}}{\overline{\left|z_{p}\right|^{2}}} \\
& =10 \log _{10} \frac{\frac{\left|y_{p}\right|^{2}}{\left|z_{p}\right|^{2}}}{} .
\end{aligned}
$$

A computer simulation was carried out according to the above-described method. The model of impulse response to be estimated for the computer simulation is made from 1,024 samples cut out arbitrarily from a sequence of pseudo-white noise. By multiplying an exponential decay curve, the impulse response is attenuated to $1 / 1,000$ in amplitude at the time corresponding to the 1,024 th sample.

The effects of the shift of the center of rectangular pulse from the sampling time on the accuracy of estimated impulse response are first examined using the ratio of the duration of shift to the sampling period. Figure 6 shows that the accuracy of the estimation decreases rapidly by the shift of the center of rectangular pulse from the sampling time. It is also found that a better estimation is achieved by the shorter pulse in the case where the center of the rectangular pulse coincides with the sampling time. Figure 7 shows the power spectra of residual signal $y_{p}$ for two cases using the ratio RPS of pulse width to the sampling period. It is recognized from this 


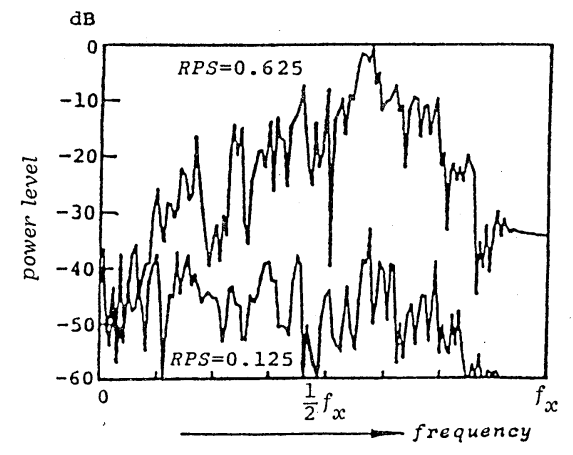

RPS : pulse width / sampling period

Fig. 7 Power spectra of the residual signal $y_{p}$.

pulse width / sampling period

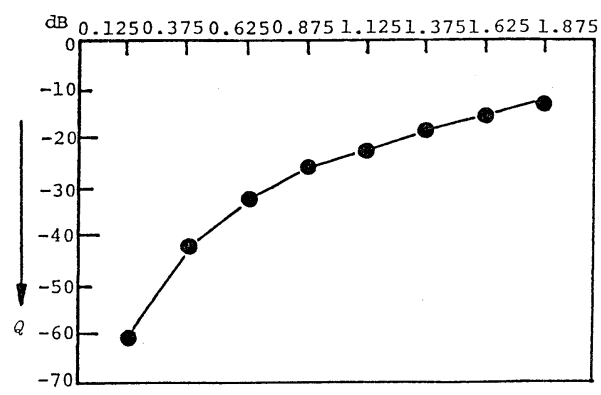

Fig. 8 Accuracy evaluation index $Q$.

figure that the accuracy deteriorates mainly in the higher frequency range. Next, the effects of the duration of source rectangular pulse was examined. Figure 8 shows the changes of the evaluation index $Q$ as a function of the ratio RPS when the center of the pulse coincides with the sampling time. Even in this case, the impulse response cannot be estimated very accurately if the duration of the rectangular pulse is long.

\section{EXPERIMENTAL VERIFICATION USING A ROOM}

Experiments were carried out in a room $3.0 \mathrm{~m} \times$ $4.2 \mathrm{~m} \times 7.2 \mathrm{~m}$ for verification of the results based on theoretical considerations and the computer simulation in the previous section. The reverberation time of the room is about $0.3 \mathrm{~s}$, and the external noise level in the room is $52 \mathrm{~dB}(\mathrm{C})$. A loudspeaker of 30 $\mathrm{cm}$ diameter and a non-directional microphone were set in the room to make a transmission system from

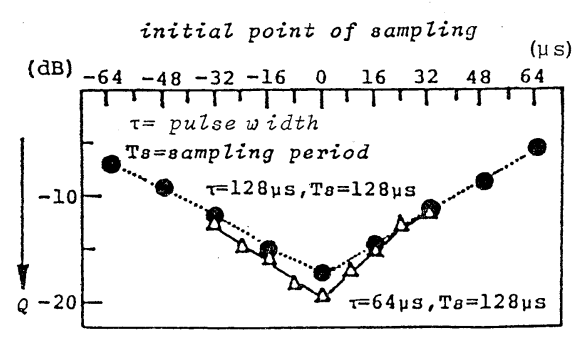

Fig. 9 Experimental results of the effects of the shift of the center of the pulse from the sampling time.

the input terminal of the driving amplifier to the output terminal of the microphone amplifier. The loudspeaker was directed upward, and the microphone was set $2 \mathrm{~m}$ apart from the loudspeaker at the same height.

To estimate the impulse response, many rectangular pulses were fed into the driving amplifier to superpose the responses for a high signal to noise ratio. The duration of each rectangular pulse used was between $16 \mu$ s and $128 \mu \mathrm{s}$. The interval between the adjacent pulses was $0.5 \mathrm{~s}$. The output of the microphone amplifier was A/D converted after having been passed through an anti-aliasing filter. To improve the signal to noise ratio, the digitalized signal was accumulated lining up the initial point of the response to the input pulse. The length of the impulse response was assumed to be less than 2,048. The number of accumulation was 100 , that is, the signal to noise ratio may have been about $20 \mathrm{~dB}$ improved. The input signal that was used for the measurement of the index $Q$ was a white noise. We measured $Q$ ten times and the average of those results is given here as the evaluation index $Q$.

The experiments were carried out in two cases. In the first case, the sampling period for the A/D conversion was $128 \mu \mathrm{s}$, and the cut off frequency of the anti-aliasing filter was $2.8 \mathrm{kHz}$. In the second case, they were $64 \mu \mathrm{s}$ and $5.6 \mathrm{kHz}$, respectively.

Figure 9 shows the effects of the shift of the center of a rectangular pulse from the sampling time on the accuracy of estimation. Results similar to those in the computer simulation were obtained. Relatively low accuracy at the point of zero time shift is considered to be caused by the room noise.

Figure 10 shows the change in the evaluation index $Q$ by the duration of source rectangular pulse. The results tend to coincide with those obtained through 


\section{D. KIM et al.: ESTIMATION OF IMPULSE RESPONSE USING A RECTANGULAR PULSE}

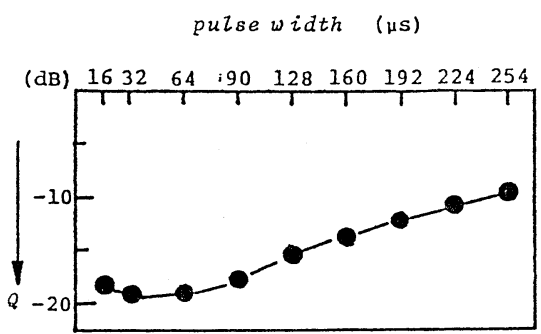

Fig. 10 Accuracy evaluation index $Q$ obtained by experiments.

computer simulation. However, the value of evaluation index $Q$ does not take a large value when the pulse width is narrow because of the presence of a large amount of external noise in the room.

\section{CONCLUSIONS}

The method to estimate the impulse response of a system using a rectangular pulse as a source signal was investigated. Since the response of the system to the rectangular pulse is used directly as the impulse response, a minimum number of computations is required for this method.

Index $Q$ is first introduced to evaluate the accuracy of the estimated impulse response of the system. This index works well even under the presence of external noises. Theoretical considerations of the method were also described in detail. It was found that the timing relation between the rectangular pulse and the $\mathrm{A} / \mathrm{D}$ conversion, as well as the pulse width and the pulse amplitude, affects the accuracy of an estimated impulse response. To evaluate the effects of the timing relation, the pulse width, and the pulse amplitude on the index $Q$, computer simulations were carried out. It was also found from experiments using a room that the index $Q$ is effective even under the presence of external noise. In the experiment using a room, the power of the difference signal between the real response and the calculated response using the estimated impulse response is less than $1 / 100$ of the real response.
The summaries of this work are follows:

1. The proposed method requires the least amount of computation, because the response of the system to the rectangular pulse is approximately equal to the impulse response of the system.

2. The effect of the duration of rectangular pulse (pulse width) on the accuracy of the estimated impulse response is evaluated by an accuracy index.

3. The response to the rectangular pulse should be multiplied by an suitable coefficient, which is determined by the amplitude and width of the rectangular pulse, and the sampling frequency.

4. The center of the source rectangular pulse should exactly coincide with the sampling time. The shift of timing causes a rapid large decrease in the accuracy.

\section{ACKNOWLEDGMENTS}

The authors wish to thank the members of the Research Center for Applied Information Sciences, Tohoku University for their kind assistance and continuous encouragement. They also wish to thank Mr. H. Koseki and Mr. M. Ono of Nippon Electric Co., Ltd. for their support in this work.

\section{REFERENCES}

1) H. Date, K. Fukudome, and T. Tanaka, "Observation of reverberation curves by musical sounds," Proc. Spring Meet. Acoust. Soc. Jpn., 751-752 (1981) (in Japanese).

2) N. Aoshima, "Computer-generated pulse signal applied for sound measurement," J. Acoust. Soc. Am. 69, 1484-1488 (1981).

3) A. Miura, R. Sato, and K. Nagata, "Subjective evaluation of delay and echo suppressors in telephone communication," 72nd Meet. A.S.A. Session 3H4 (1966).

4) W. A. Clark, R. M. Brown, M. H. Goldstein, Jr., C. E. Molnar, D. F. O'Brien, and H. E. Zieman, "The average response computer (ARC): A digital device for computing averages and amplitude and time histograms of electrophysiological response," IRE BME8, 46-51 (1961). 